\title{
Gliomatosis Cerebri Type II
}

National Cancer Institute

\section{Source}

National Cancer Institute. Gliomatosis Cerebri Type II. NCI Thesaurus. Code C41843.

A classic form of gliomatosis cerebri. It is characterized by diffuse growth of neoplastic glial tissue without any focal mass. 